\title{
BIOGENETICALLY INSPIRED SYNTHESIS OF LINGZHIOL
}

\author{
Krishna Sharmah Gautam and Vladimir B. Birman* \\ Department of Chemistry, Washington University, Campus Box 1134, One Brookings Drive, \\ Saint Louis, Missouri 63130
}

SUPPORTING INFORMATION

\section{GENERAL.}

All reagents were obtained commercially and used as received unless specified otherwise. All reactions were carried out under argon, unless noted otherwise. Dichloromethane and acetonitrile used as reaction media were freshly distilled from calcium hydride. Solvents used for chromatography were ACS or HPLC grade. Reactions were monitored by thin layer chromatography (TLC) and by ${ }^{1} \mathrm{H}$ NMR. Uniplate HLF $(250 \mu \mathrm{m})$ silica gel plates were used for TLC analyses. Flash column chromatography was performed over Sorbent Technologies silica gel (40-63 mm). ${ }^{1} \mathrm{H}$ and ${ }^{13} \mathrm{C}$ NMR spectra were recorded on a Mercury $300 \mathrm{MHz}$ and DD2 $500 \mathrm{MHz}$ Agilent spectrometer. The chemical shifts are reported as $\delta$ values (ppm) relative to TMS using the residual $\mathrm{CHCl}_{3}$ peak $(7.26 \mathrm{ppm})$ as the reference. Melting points were measured on a Stuart SMP10 melting point apparatus. High-Resolution mass spectral analyses were performed at Washington University MS Center on a Bruker MaXis QTOF mass spectrometer using Electrospray Ionization (ESI) method. ${ }^{1}$ Infrared spectra were recorded on a Bruker Alpha Platinum-ATR. HPLC analyses were performed on a Shimadzu LC system using a Chiralcel OD$\mathrm{H}$ analytical chiral stationary phase column $(4.6 \times 250 \mathrm{~mm}$, Chiral Technologies, Inc.) and hexane/isopropanol (95:5) eluent at $1 \mathrm{~mL} / \mathrm{min}$ flow rate. Signs of optical rotation were determined on a Rudolph Autopol III polarimeter.

\section{Synthetic Procedures}<smiles>COc1ccc(OC)c2c1CCC(C(C)=O)C2=O</smiles>

( \pm )-Diketone 14. Ketoester 13 was prepared according to a published procedure ${ }^{2}$ from 5,8 dimethoxytetralone $2 .{ }^{3}$ To a solution of $13(529 \mathrm{mg}, 2.00 \mathrm{mmol})$ in $11 \mathrm{~mL}$ of dichloromethane stirring under argon, 1,1,3,3-tetramethylguanidine was added $(50 \mu \mathrm{L}, 46 \mathrm{mg}, 0.40 \mathrm{mmol})$, followed by methyl vinyl ketone $(416 \mu \mathrm{L}, 358 \mathrm{mg}, 5.10 \mathrm{mmol})$. The mixture was stirred at room temperature for 16 hours, then concentrated down to ca. $1 \mathrm{~mL}$, loaded onto a silica gel column, and eluted with hexane/ethyl acetate (3:2) to give the product as yellow oil (531 mg, 79\% yield). ${ }^{1} \mathbf{H}$ NMR (300 $\left.\mathrm{MHz} \mathrm{CDCl}_{3}\right): \delta 6.95(\mathrm{~d}, J=9.4 \mathrm{~Hz}, 1 \mathrm{H}), 6.79(\mathrm{~d}, J=9.4 \mathrm{~Hz}, 1 \mathrm{H}), 3.85(\mathrm{~s}, 3 \mathrm{H}), 3.80(\mathrm{~s}, 3 \mathrm{H}), 3.64$ (s, 3H), 2.98-2.67 (m, 3H), 2.57-2.47 (m, 2H), 2.25-2.15 (m, 1H), 2.14 (s, 3H), 2.11-1.94 (m, 2H). ${ }^{13} \mathrm{C}$ NMR (300 MHz, $\left.\mathrm{CDCl}_{3}\right)$ : 206.9, 194.0, 171.7, 153.4, 149.5, 132.3, 121.9, 114.6, 109.8, 56.7, 
55.7, 55.1, 51.6, 34.4, 29.6, 29.2, 27.1, 20.0. IR $\left(\mathrm{cm}^{-1}\right): 2936,2835,1715,1683,1586,1476,1349$, 1260, 1196, 1068, 982, 806, 718. MS: HR-ESI calculated for $\left[\mathrm{C}_{18} \mathrm{H}_{22} \mathrm{O}_{6}+\mathrm{H}\right]^{+}: 335.1489$, found: 335.1490 .
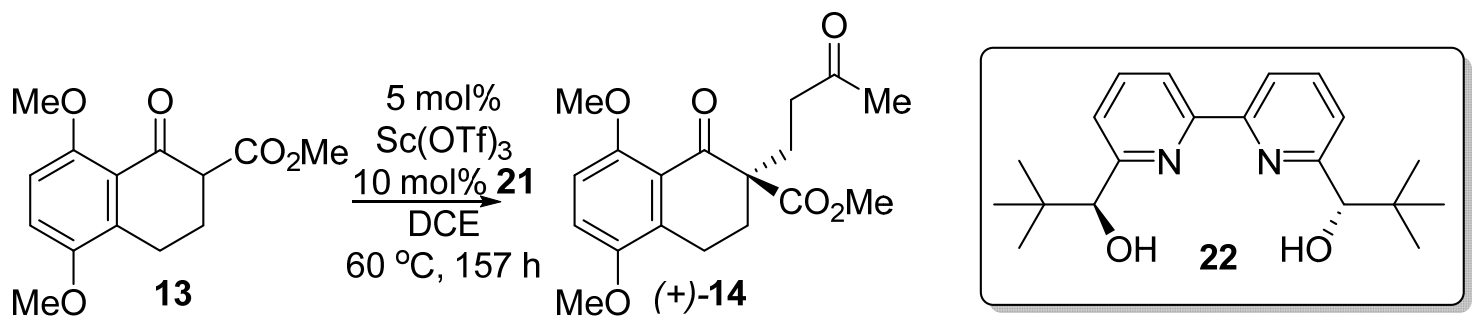

Asymmetric synthesis of diketone $(R)-14 .^{4}$ A solution of scandium triflate $(18.0 \mathrm{mg}, 0.036 \mathrm{mmol})$ and Bolm's ligand $(S, S)-22^{5}(23.5 \mathrm{mg}, 0.072 \mathrm{mmol})$ in $5.0 \mathrm{~mL}$ of anhydrous 1,2-dichloroethane was heated under argon at $60^{\circ} \mathrm{C}$ for 1 hour, diluted with an additional $23 \mathrm{~mL}$ of 1,2-dichloroethane and heated at the same temperature for another 20 minutes. A solution of ketoester 13 (190 mg, $0.722 \mathrm{mmol})$ in $5 \mathrm{~mL}$ of 1,2-dichloroethane and methyl vinyl ketone $(120 \mu \mathrm{L}, 1.44 \mathrm{mmol})$ in $3 \mathrm{~mL}$ of 1,2-dichloroethane were added consecutively to the resulting scandium complex solution. The reaction mixture was stirred at $60{ }^{\circ} \mathrm{C}$ for 168 hours, then concentrated to ca. $1 \mathrm{~mL}$ and loaded onto a silica gel column and eluted with hexane/ethyl acetate $(5: 1 \rightarrow 3: 1)$ to afford the unreacted starting material $(104 \mathrm{mg}, 0.393 \mathrm{mmol}$, $54 \%$ recovery) followed by the product as a yellow oil $(80 \mathrm{mg}$, $34 \%$ yield). The recovered starting material was re-subjected to the same protocol to deliver an additional $43 \mathrm{mg}$ of $(R)-14(33 \%$ yield) and $58 \mathrm{mg}$ of unreacted 13 (56\% recovery). The combined yield from 2 cycles was $51 \%$, or $74 \%$ based on recovered starting material. HPLC analysis indicated $93.5 \%$ ee. HPLC: $(5 \%$ isopropanol/hexane) $[72.3 \mathrm{~min}$ (minor); $79.1 \mathrm{~min}$ (major $] .[\alpha]_{\mathrm{D}}{ }^{26}=$ $+30.8^{\circ}\left(\mathrm{c}=5.8, \mathrm{CHCl}_{3}\right)$. The absolute stereochemistry of intermediates 14-16, 20 and 21was assigned by correlation of the optical rotation of the known intermediate $(+)-5$ (vide infra) with

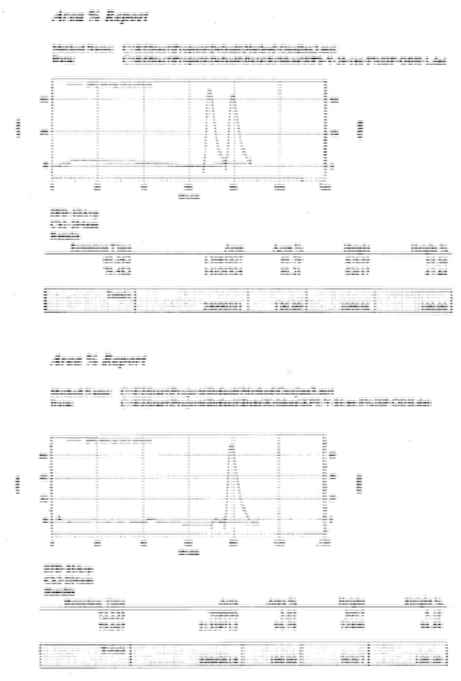
previously reported data. ${ }^{6}$<smiles>COc1ccc(OC)c2c1CC[C@](CCC(C)=O)(OC)C2=O</smiles>

$\mathrm{NaOMe}, \mathrm{MeOH}$ $(79 \%)$<smiles>COc1ccc(OC)c2c1CCC1(OC)CCC(=O)C=C21</smiles>

Tricyclic ketone 15. A sodium methoxide solution freshly prepared from sodium (271 mg, 11.8 mg-atom) and $16 \mathrm{~mL}$ of anhydrous methanol was cooled to room temperature. A solution of diketone $( \pm)-14(530 \mathrm{mg}, 1.59 \mathrm{mmol})$ in $9 \mathrm{~mL}$ of methanol was added to it dropwise over 10 minutes with stirring. When the reaction was complete by TLC (13 hours), the mixture was rotary evaporated to dryness, treated with water and extracted multiple times with ethyl acetate. The organic phase was dried over $\mathrm{MgSO}_{4}$, and rotary evaporated to give the product as a yellow oil, 
which crystallized on standing into a pale-yellow solid (396 mg, 79\% yield). ${ }^{1}$ H NMR (300 MHz, $\left.\mathrm{CDCl}_{3}\right): \delta 7.28(\mathrm{~s}, 1 \mathrm{H}), 6.80$ and 6.78 (AB system, $J=9.1 \mathrm{~Hz}, 2 \mathrm{H}), 3.81$ (s, 3H), 3.78 (s, 3H), 3.61 (s, $3 \mathrm{H}), 2.92-2.86(\mathrm{~m}, 1 \mathrm{H}), 2.70-2.55(\mathrm{~m}, 1 \mathrm{H}), 2.50-$ $2.40(\mathrm{~m}, 4 \mathrm{H}), 2.11-2.00(\mathrm{~m}, 1 \mathrm{H}), 1.84-1.73(\mathrm{~m}$, 1H). ${ }^{13} \mathrm{C}$ NMR (300 MHz, $\left.\mathrm{CDCl}_{3}\right): 200.3,174.1$, $153.4,151.8,150.8,129.0,128.8,122.9,111.6$, 109.9, 56.1, 55.1, 52.6, 47.7, 34.5, 34.5, 34.0, 21.5. IR $\left(\mathrm{cm}^{-1}\right): 2951,2842,1725,2655,1573,1465$, 1261, 1199, 1071, 802, 732 MS: HR-ESI calculated for $\left[\mathrm{C}_{18} \mathrm{H}_{20} \mathrm{O}_{5}+\mathrm{H}\right]^{+}$: 317.1384, found: 317.1384 . mp $144-147^{\circ} \mathrm{C}$.

Following the same protocol, enantioenriched $(R)$ $\mathbf{1 4}$ (80 mg, 93.5\% ee) was converted into (R)-15 (60 $\mathrm{mg}, 79 \%$ yield) with no appreciable deterioration of the enantiomeric excess (92\% ee). HPLC (3.3\% isopropanol/hexane): $52.0 \mathrm{~min}$ (minor); $59.3 \mathrm{~min}$ (major); $[\alpha]^{23} \mathrm{D}=+274.5^{\circ}\left(\mathrm{c}=1.3, \mathrm{CHCl}_{3}\right)$.<smiles>COc1ccc(OC)c2c1CC[C@]1(C(C)=O)CCC(=O)C=C21</smiles>

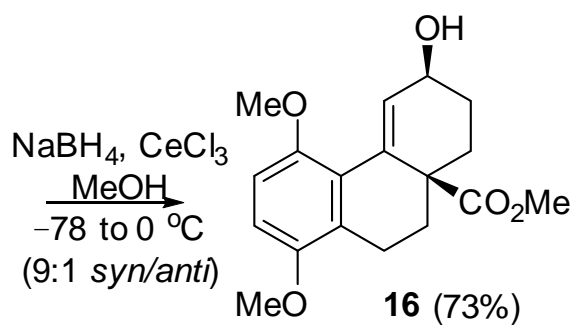

( \pm -Cis-Allylic alcohol 16. To a solution of ketone $( \pm)-15(100 \mathrm{mg}, 0.316 \mathrm{mmol})$ in $10 \mathrm{~mL}$ of methanol cooled to $-78{ }^{\circ} \mathrm{C}, \mathrm{CeCl}_{3} \cdot 7 \mathrm{H}_{2} \mathrm{O}(130 \mathrm{mg}, 0.35 \mathrm{mmol})$ was added, followed by $\mathrm{NaBH}_{4}(60.5 \mathrm{mg}, 1.6$ mmol). The reaction was allowed to warm up to $0{ }^{\circ} \mathrm{C}$. After completion of the reaction, the mixture was rotary evaporated to dryness, treated with water and extracted multiple times with ethyl acetate. The organic phase was concentrated down to about $1 \mathrm{~mL}$ and loaded onto a silica gel column. Elution with hexane/ethyl acetate (1:1) provided the minor trans-diastereomer (colorless oil, $8.6 \mathrm{mg}$, $8.6 \%$ yield) followed by the desired cisdiastereomer 16 (white solid, $73 \mathrm{mg}, 73 \%$ yield). Data for the cis-isomer 16: ${ }^{1} \mathbf{H}$ NMR $(300 \mathrm{MHz}$, $\left.\mathrm{CDCl}_{3}\right): \delta 6.90(\mathrm{~s}, 1 \mathrm{H}), 6.76$ and 6.65 (AB system, $J=9.1 \mathrm{~Hz}, 2 \mathrm{H}), 4.34$ (broad, 1H), 3.79 (s, 3H), 3.76 $(\mathrm{s}, 3 \mathrm{H}), 3.57(\mathrm{~s}, 3 \mathrm{H}), 2.87-2.79(\mathrm{~m}, 1 \mathrm{H}), 2.65-2.52$ $(\mathrm{m}, 1 \mathrm{H}), 2.42-2.36(\mathrm{~m}, 1 \mathrm{H}), 2.30-2.25(\mathrm{~m}, 1 \mathrm{H})$,

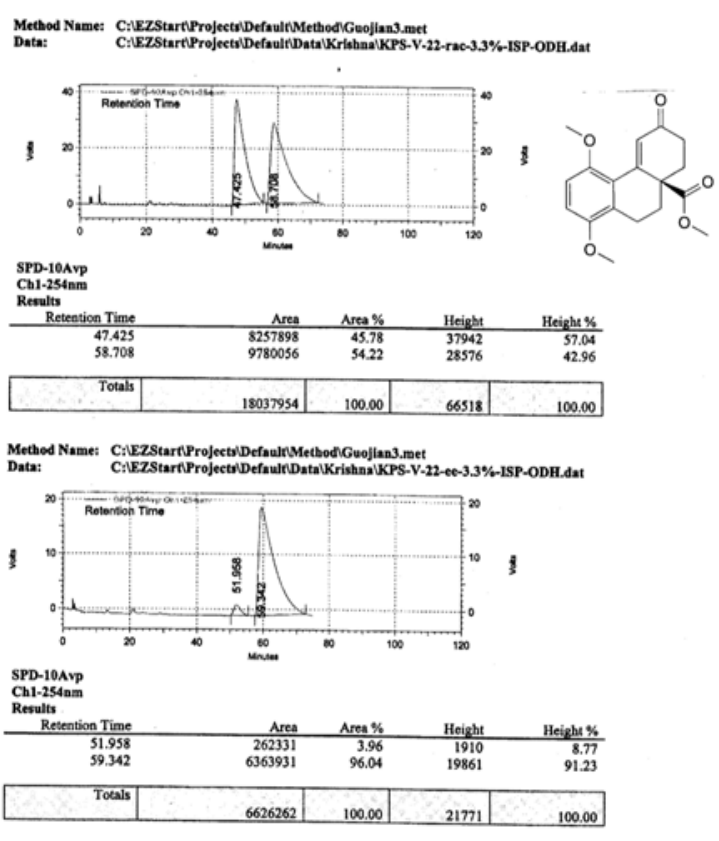

$16(73 \%)$
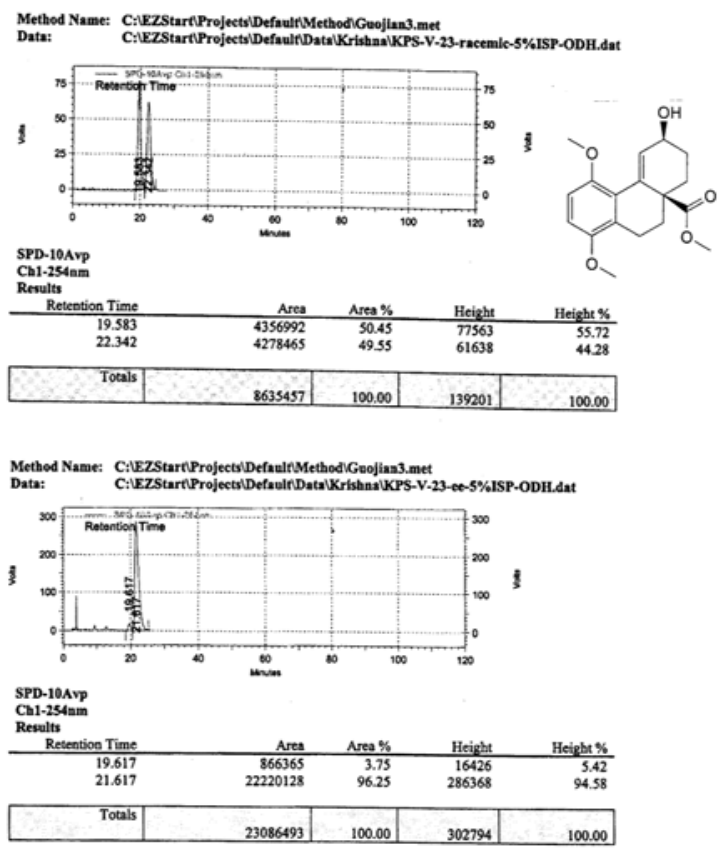
2.07-2.00 (m, 1H), 1.71-1.60 (m, 4H). ${ }^{13} \mathrm{C}$ NMR (300 MHz, $\left.\mathrm{CDCl}_{3}\right): 176.0,151.8,151.2,132.6$, $132.5,126.5,125.3,110.5,108.25,68.0,56.4,55.5,52.1,46.6,34.1,33.5,28.7,21.6$. IR $\left(\mathrm{cm}^{-1}\right)$ : 3410, 2942, 2867, 1727, 1596, 1474, 1248, 1172, 1136, 1072, 797, 731. MS: HR-ESI calculated for $\left[\mathrm{C}_{18} \mathrm{H}_{22} \mathrm{O}_{5}+\mathrm{K}\right]^{+}: 357.1099$, found: 357.1099 . mp $146-148{ }^{\circ} \mathrm{C}$.

Following the same protocol, enantioenriched $(R)-\mathbf{1 5}(57 \mathrm{mg})$ was converted into (+)-16 (43 mg, $73 \%$ yield). HPLC (5\% isopropanol/hexane): $19.6 \mathrm{~min}$ (minor); $21.6 \mathrm{~min}$ (major); $[\alpha]_{\mathrm{D}}^{23}=$ $+127.5^{\circ}\left(\mathrm{c}=0.65, \mathrm{CHCl}_{3}\right)$.<smiles>COc1ccc(OC)c2c1CC[C@@]1(C(C)=O)CC[C@@H](O)C=C21</smiles>

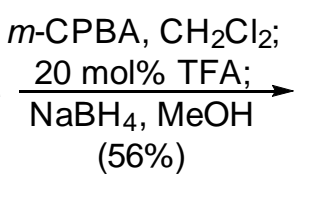

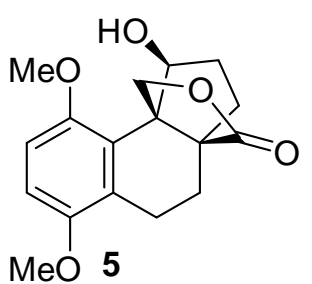<smiles>COc1ccc(OC)c2c1CC[C@]1(CO)[C@@H](C)CC[C@@]21C(C)(C)C</smiles>

( \pm )-Lactone 5. To a solution of alcohol $16(51 \mathrm{mg}, 0.158 \mathrm{mmol})$ in $8 \mathrm{~mL}$ of dichloromethane stirring at $0{ }^{\circ} \mathrm{C}, \mathrm{m}$-CPBA (46 mg, $0.24 \mathrm{mmol}$ ) was added. After 20 minutes of stirring, trifluoroacetic acid $(2.4 \mu \mathrm{L}, 3.6 \mathrm{mg}, 0.031 \mathrm{mmol}, 20 \mathrm{~mol} \%)$ was added, followed, in 30 seconds, by addition of saturated aqueous solution of $\mathrm{NaHCO}_{3}$. After solvent removal, the residue was dissolved in $5 \mathrm{~mL}$ of methanol, cooled to $0{ }^{\circ} \mathrm{C}$, treated with $\mathrm{NaBH}_{4}(33 \mathrm{mg}, 0.78 \mathrm{mmol})$, and stirred overnight. The reaction mixture was rotary evaporated to dryness, treated with water and extracted with $\mathrm{CH}_{2} \mathrm{Cl}_{2}(2 \times 5 \mathrm{~mL})$. The organic extract was concentrated down and chromatographed using hexane/ethyl acetate eluent $(3: 1 \rightarrow 1: 1)$ to obtain $( \pm)-5$ as white solid $(27 \mathrm{mg}, 56 \%$ yield $)$. mp 183 $185^{\circ} \mathrm{C} .{ }^{1} \mathrm{H}$ NMR data for 5 matched those reported in the literature. ${ }^{6}$

Following the same protocol, enantioenriched (+)-16 (40 mg) was converted into (+)-5 (22 mg, $58 \%$ yield, $92.0 \%$ ee). HPLC $(5 \%$ isopropanol/hexane): [46.8 $\mathrm{min}$ (minor); 65.4 $\min$ (major]. $[\alpha] \mathrm{D}^{23}=+71^{\circ}(\mathrm{c}=0.2, \mathrm{MeOH})$. The absolute configuration was assigned by comparison of the sign of optical rotation with previously reported data. ${ }^{6}$ In addition, $7.5 \mathrm{mg}$ of anti-aldol 18a was isolated (18\% yield). Data for 18a: ${ }^{1} \mathbf{H}$ NMR $\left(300 \mathrm{MHz}, \mathrm{CDCl}_{3}\right): \delta$ $9.62(\mathrm{~s}, 1 \mathrm{H}), 6.78-6.74(\mathrm{~m}, 2 \mathrm{H}), 4.83(\mathrm{~m}, 1 \mathrm{H})$, $3.78(\mathrm{~s}, 3 \mathrm{H}), 3.77(\mathrm{~s}, 3 \mathrm{H}), 3.61(\mathrm{~s}, 3 \mathrm{H}), 3.00-$ $2.90(\mathrm{~m}, 1 \mathrm{H}), 2.57-2.45(\mathrm{~m}, 1 \mathrm{H}), 2.36-2.24(\mathrm{~m}$, $2 \mathrm{H}), 2.19-2.10(\mathrm{~m}, 1 \mathrm{H}), 2.06-1.96(\mathrm{~m}, 2 \mathrm{H})$, 1.92-1.80 (m, 1H). ${ }^{13} \mathrm{C}$ NMR $(600 \mathrm{MHz}$, CDCl3): 206.8, 175.5, 152.0, 150.5, 129.3, 123.6, 109.3, 108.6, 77.6, 65.2, 59.4, 56.1, 55.8, 52.3, 33.8, 32.0, 29.4, 21.3. IR $\left(\mathrm{cm}^{-1}\right)$ : 3430, 2947, 2842, 1722, 1658, 1474, 1450, 1258, 1173, 1069, 796, 729. MS: HR-ESI calculated for $[\mathrm{C} 18 \mathrm{H} 22 \mathrm{O} 6+\mathrm{H}]^{+}$: 335.1477 , found: 335.1489 .

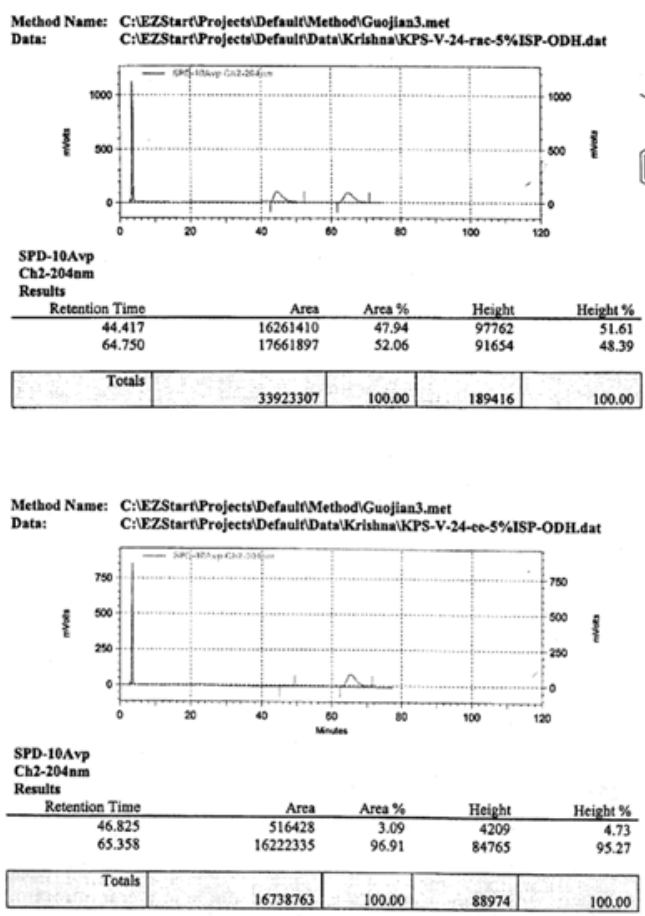



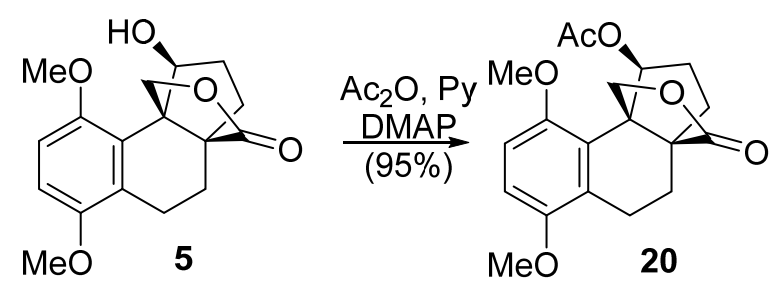

( \pm )-Acetate 20. A solution of $( \pm)-5(27 \mathrm{mg})$ in $1 \mathrm{~mL}$ of $\mathrm{CH}_{2} \mathrm{Cl}_{2}$ stirring at $0{ }^{\circ} \mathrm{C}$ was treated successively with acetic anhydride $(25.3 \mu \mathrm{L}, 0.27 \mathrm{mmol})$, pyridine $(21.7 \mu \mathrm{L}, 0.27 \mathrm{mmol})$ and DMAP ( $2 \mathrm{mg}, 0.018 \mathrm{mmol}$ ), allowed to warm to room temperature and stirred overnight. The reaction mixture was diluted with $\mathrm{CH}_{2} \mathrm{Cl}_{2}$, washed with $1 \mathrm{M} \mathrm{HCl}$ and then water, dried over $\mathrm{MgSO}_{4}$, and rotary evaporated to obtain ( \pm )-20 as white solid (28 mg, $0.084 \mathrm{mmol}, 95 \%$ yield). ${ }^{1} \mathbf{H}$ NMR $\left(300 \mathrm{MHz}, \mathrm{CDCl}_{3}\right): \delta 6.72$ and $6.69(\mathrm{AB}$ system, $J=9.4 \mathrm{~Hz}, 2 \mathrm{H}), 5.42(\mathrm{~s}, 1 \mathrm{H}), 4.91(\mathrm{~d}, J=10.0$ $\mathrm{Hz}, 1 \mathrm{H}), 4.09(\mathrm{~J}=10.0 \mathrm{~Hz}, 1 \mathrm{H}), 3.79(\mathrm{~s}, 3 \mathrm{H}), 3.77(\mathrm{~s}, 3 \mathrm{H}), 3.13-3.06(\mathrm{~m}, 1 \mathrm{H}), 2.50-2.31(\mathrm{~m}, 2 \mathrm{H})$, 2.22-2.18 (m, 1H), $2.12(\mathrm{~s}, 3 \mathrm{H}), 2.04-1.94(\mathrm{~m}, 1 \mathrm{H}), 1.78-1.60(\mathrm{~m}, 3 \mathrm{H}) .{ }^{13} \mathrm{C}$ NMR $(300 \mathrm{MHz}$, $\left.\mathrm{CDCl}_{3}\right): 182.7,170.4,151.5,150.9,127.3,125.8,109.1,108.5,82.8,72.4,55.9,55.6,55.0,51.7$, 32.9, 31.5, 27.6, 231.5, 18.5. IR $\left(\mathrm{cm}^{-1}\right): 2939,2801,1764,1737,1477,1370,1232,1175,1113$, 1086, 1020, 990, 800, 713. MS: HR-ESI calculated for $\left[2 \mathrm{C}_{19} \mathrm{H}_{22} \mathrm{O}_{6}+\mathrm{Na}\right]^{+}: 715.2725$, found: 715.2749. mp $178-181^{\circ} \mathrm{C}$.

Following the same protocol, enantioenriched (+)-5 (20 mg) was converted into (+)-20 (22 mg, $96 \%$ yield $) .[\alpha]_{D^{26}}=+75^{\circ}(\mathrm{c}=0.1, \mathrm{MeOH})$.
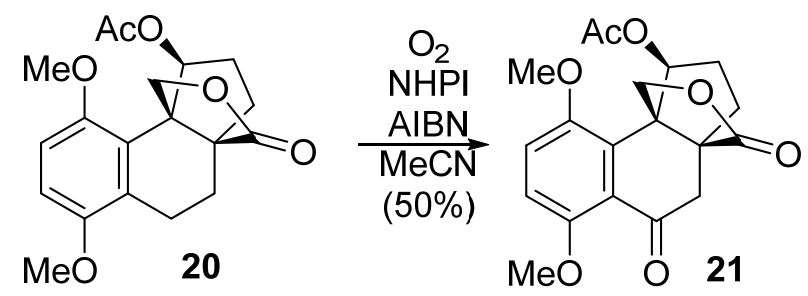

$( \pm)$-O,O-Dimethyl-lingzhiol acetate $21 .{ }^{7}$ An oven-dried flask was charged with acetate $( \pm)$-20 (24 $\mathrm{mg}, 0.072 \mathrm{mmol}$ ), N-hydroxyphthalimide (2.3 mg, $0.014 \mathrm{mmol}, 20 \mathrm{~mol} \%$ ), AIBN (1.2 mg, 0.0072 $\mathrm{mmol}, 10 \mathrm{~mol} \%$ ), and $1 \mathrm{~mL}$ of acetonitrile, equipped with a reflux condenser, immersed into an oil bath preheated with $70^{\circ} \mathrm{C}$ and kept at that temperature for 24 hours, while oxygen was bubbled continuously through the reaction mixture by means of a long steel needle. The reaction mixture was allowed to cool to room temperature, loaded directly onto a silica gel column and eluted with hexane/ethyl acetate (1:1) to obtain ( \pm )-21 as white solid (13 mg, 50\% yield). ${ }^{1} \mathbf{H}$ NMR (300 MHz, $\mathrm{CDCl}_{3}$ ): $\delta 7.08(\mathrm{~d}, J=8.8 \mathrm{~Hz}, 1 \mathrm{H}), 6.93(\mathrm{~d}, J=8.8 \mathrm{~Hz}, 1 \mathrm{H}), 5.48$ (broad, $\left.1 \mathrm{H}\right), 4.99$ (d, $J=10.5$ $\mathrm{Hz}, 1 \mathrm{H}), 4.29(\mathrm{~d}, J=10.5 \mathrm{~Hz}, 1 \mathrm{H}), 3.85(\mathrm{~s}, 3 \mathrm{H}), 3.83(\mathrm{~s}, 3 \mathrm{H}), 2.87$ and 2.80 (AB system, $J=12.9$ $\mathrm{Hz}, 2 \mathrm{H}), 2.55-2.44(\mathrm{~m}, 1 \mathrm{H}), 2.13(\mathrm{~s}, 3 \mathrm{H}), 2.00-1.92(\mathrm{~m}, 1 \mathrm{H}), 1.75-1.64(\mathrm{~m}, 2 \mathrm{H}) .{ }^{13} \mathrm{C}$ NMR $(300$ $\left.\mathrm{MHz}, \mathrm{CDCl}_{3}\right)$ : 194.1, 179.4, 170.0, 152.3, 150.3, 130.2, 123.0, 117.0, 112.5, 81.6, 71.1, 56.5, 55.9, 55.2, 52.8, 44.3, 31.8, 31.5, 21.3. IR $\left(\mathrm{cm}^{-1}\right): 2950,2817,1726,1655,1574,1473,1261,1071$, $1018,803,731$. MS: HR-ESI calculated for $\left[\mathrm{C}_{19} \mathrm{H}_{20} \mathrm{O} 7+\mathrm{H}\right]^{+}: 361.1266$, found: 361.1282 . mp 198$201{ }^{\circ} \mathrm{C}$.

Following the same protocol, enantioenriched $(+)-20(19.5 \mathrm{mg})$ was converted into $(+)-21(11 \mathrm{mg}$, $51 \%$ yield $) .[\alpha]_{\mathrm{D}}^{25}=+119.3^{\circ}(\mathrm{c}=0.19, \mathrm{MeOH})$. 


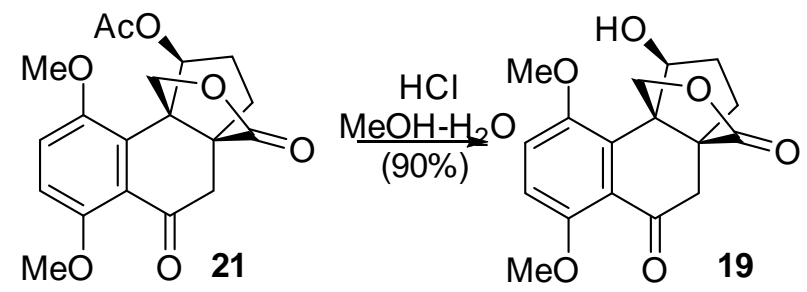

$( \pm)-O, O$-Dimethyl-lingzhiol 19. To a solution of $( \pm)-21(13 \mathrm{mg}, 0.035 \mathrm{mmol})$ in $3 \mathrm{~mL}$ of methanol, $1.6 \mathrm{~mL}$ of $3 \mathrm{M}$ aqueous $\mathrm{HCl}$ was added. The mixture was refluxed for 15 hours, allowed to cool and concentrated down. The evaporation residue was taken up in $\mathrm{CH}_{2} \mathrm{Cl}_{2}$, washed with water, dried over $\mathrm{MgSO}_{4}$, and rotary evaporated to afford ( \pm )-19 as colorless solid (10.5 $\mathrm{mg}, 0.0315 \mathrm{mmol}, 90 \%$ yield). mp 205-207 ${ }^{\circ} \mathrm{C}$ (dec). ${ }^{1} \mathrm{H}$ NMR data for 19 matched those reported in the literature. ${ }^{6}$

Following the same protocol, enantioenriched (+)-21 (8 mg) was converted into (+)-19 (6 mg, 92\% yield). $[\alpha]_{\mathrm{D}}^{24}=+27.1^{\circ}(\mathrm{c}=0.42, \mathrm{MeOH})$.<smiles>COc1ccc(OC)c2c1C(=O)CC13C(=O)CCC(O)(CCC1=O)C23C</smiles>
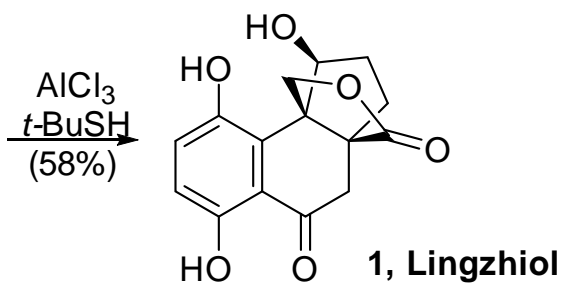

( \pm )-Lingzhiol 1. O,O-Dimethyl-lingzhiol $( \pm)$-19 (10 mg, $0.03 \mathrm{mmol})$ was treated with anhydrous $\mathrm{AlCl}_{3}$ and tertbutylmercaptan according to the procedure of Long, Huang and co-workers. ${ }^{6}$ Racemic lingzhiol ( \pm )-1 was obtained as a yellow solid (5.0 $\mathrm{mg}, 0.017 \mathrm{mmol}, 58 \%$ yield). mp $213-215^{\circ} \mathrm{C}$ (dec). ${ }^{1} \mathrm{H}$ NMR data for 1 matched those reported in the literature. ${ }^{6}$

Following the same protocol, enantioenriched $(+)-19(3.4$ $\mathrm{mg})$ was converted into $(+)-1(1.8 \mathrm{mg}, 60 \%$ yield, $93.5 \%$ ee). HPLC (20\% isopropanol/hexane): $9.7 \mathrm{~min}$ (minor); $12.7 \mathrm{~min}$ (major).

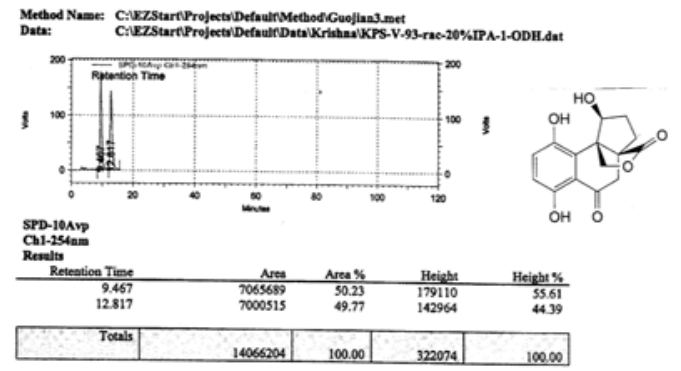

\section{REFERENCES}

${ }^{1}$ The mass spectrometry facility was supported by

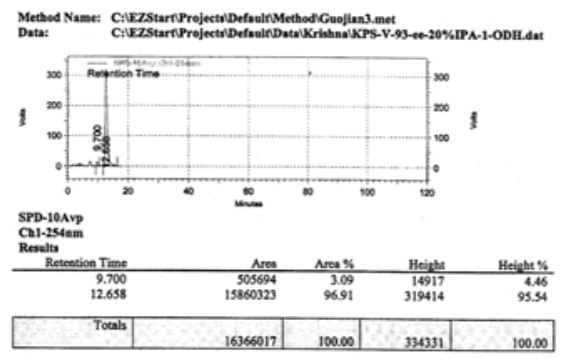
grants from the National Institute of General Medical Sciences (8 P41 GM103422).

${ }^{2}$ Johnson, D. W.; Mander, L. N. Aust. J. Chem. 1978, 31, 1561.

${ }^{3}$ The starting 5,8-dimethoxytetralone 2 is commercially available, but expensive. It can be prepared via a published procedure: Wipf, P.; Jung, J-K. J. Org. Chem.,2000, 65, 6319.

${ }^{4}$ Ogawa, C.; Kizu, K.; Shimizu, H.; Takeuchi, M.; Kobayashi, S., Chem. Asian J. 2006, 1, 121.

${ }^{5}$ Bolm, C.; Ewald, M.; Felder, M.; Schlingloff, G., Chem. Ber. 1992, 125, 1169.

${ }^{6}$ Long, R.; Huang, J.; Shao, W.; Liu, S.; Lan, Y.; Gong, J.; Yang, Z. Nat. Commun. 2014, 5, 5707.

${ }^{7}$ Fukuda, O.; Sakaguchi, S.; Ishii, Y., Adv. Synth. Catal. 2001, 343, 809. 


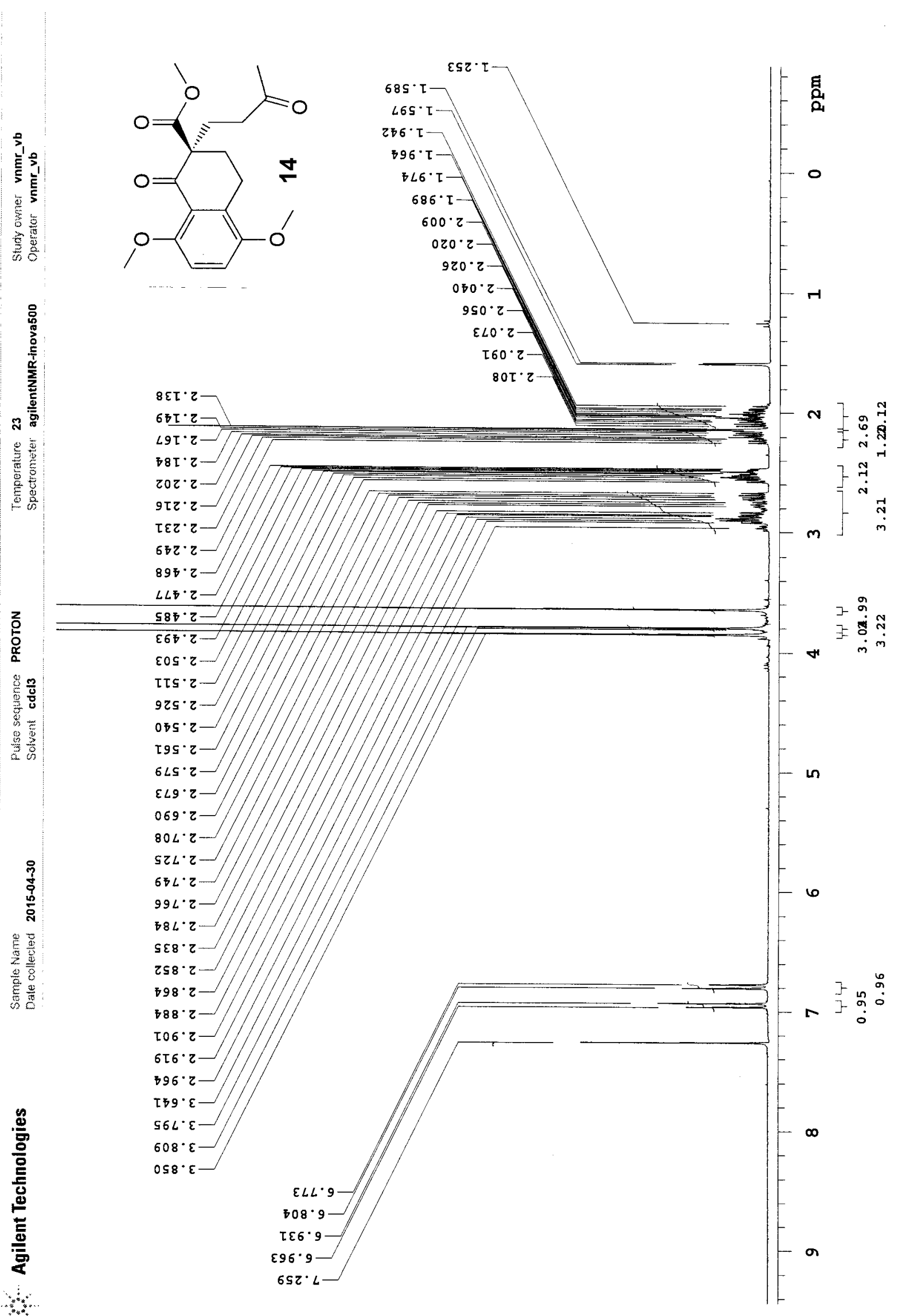




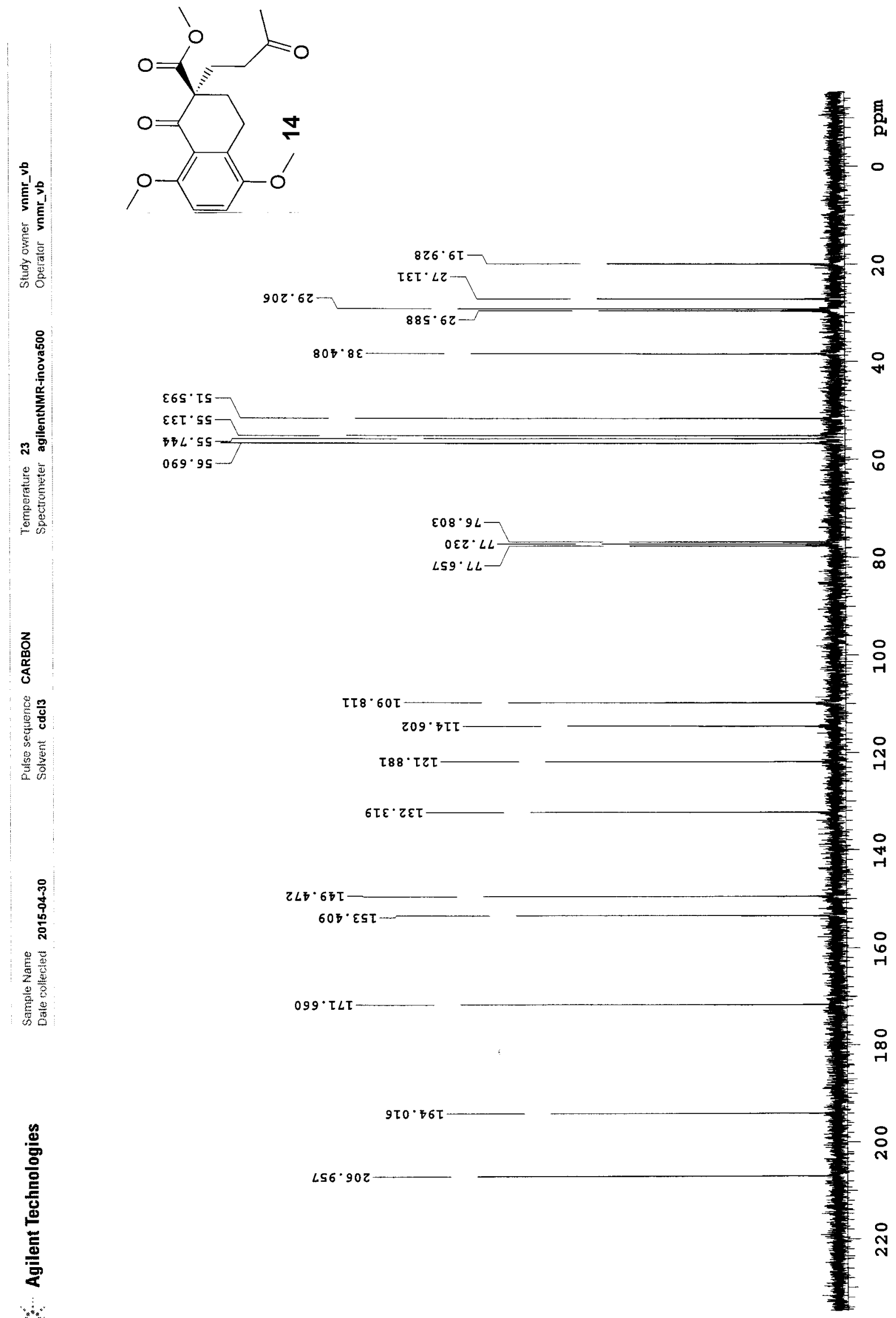

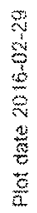

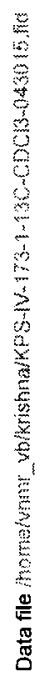

S-8 


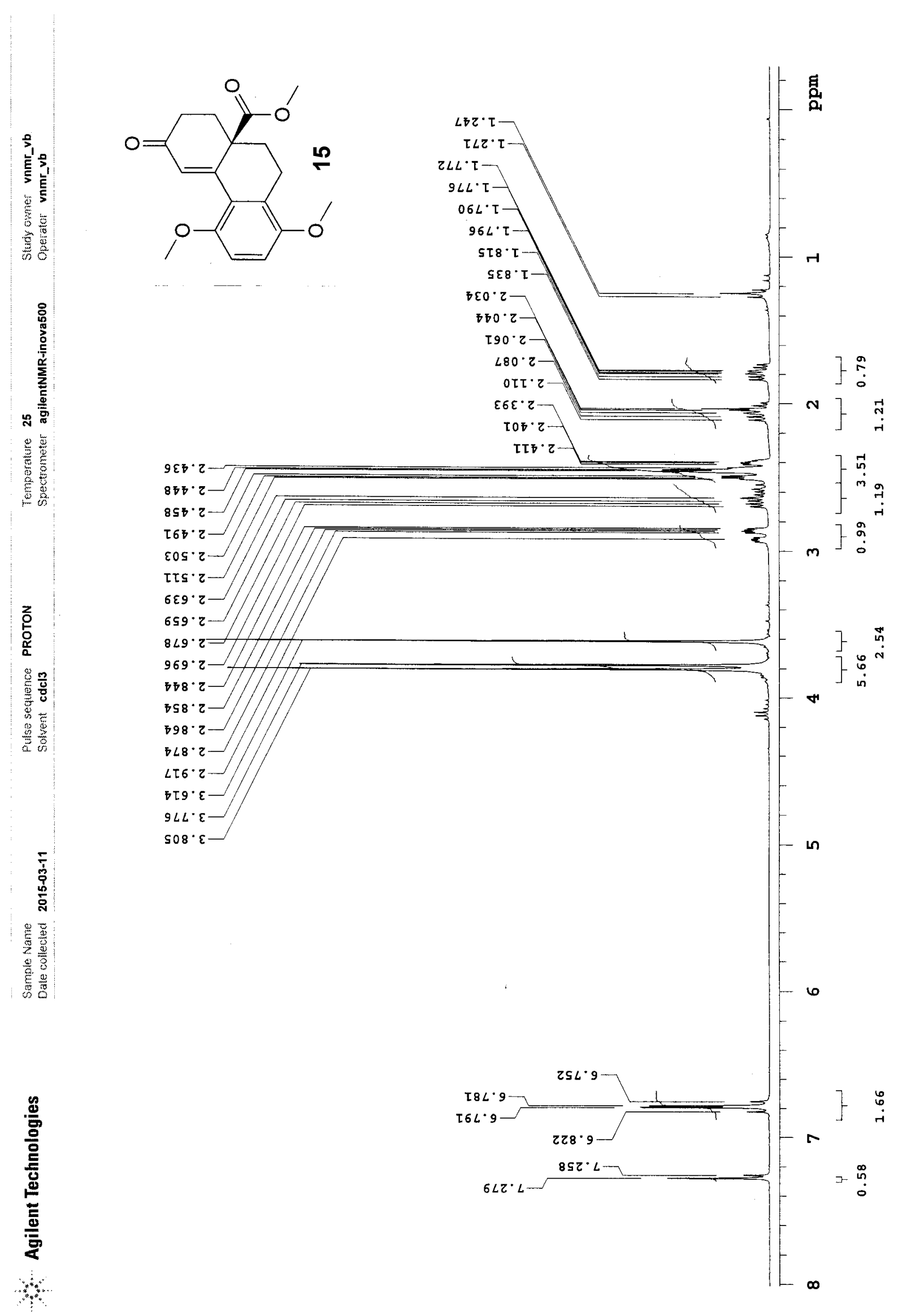



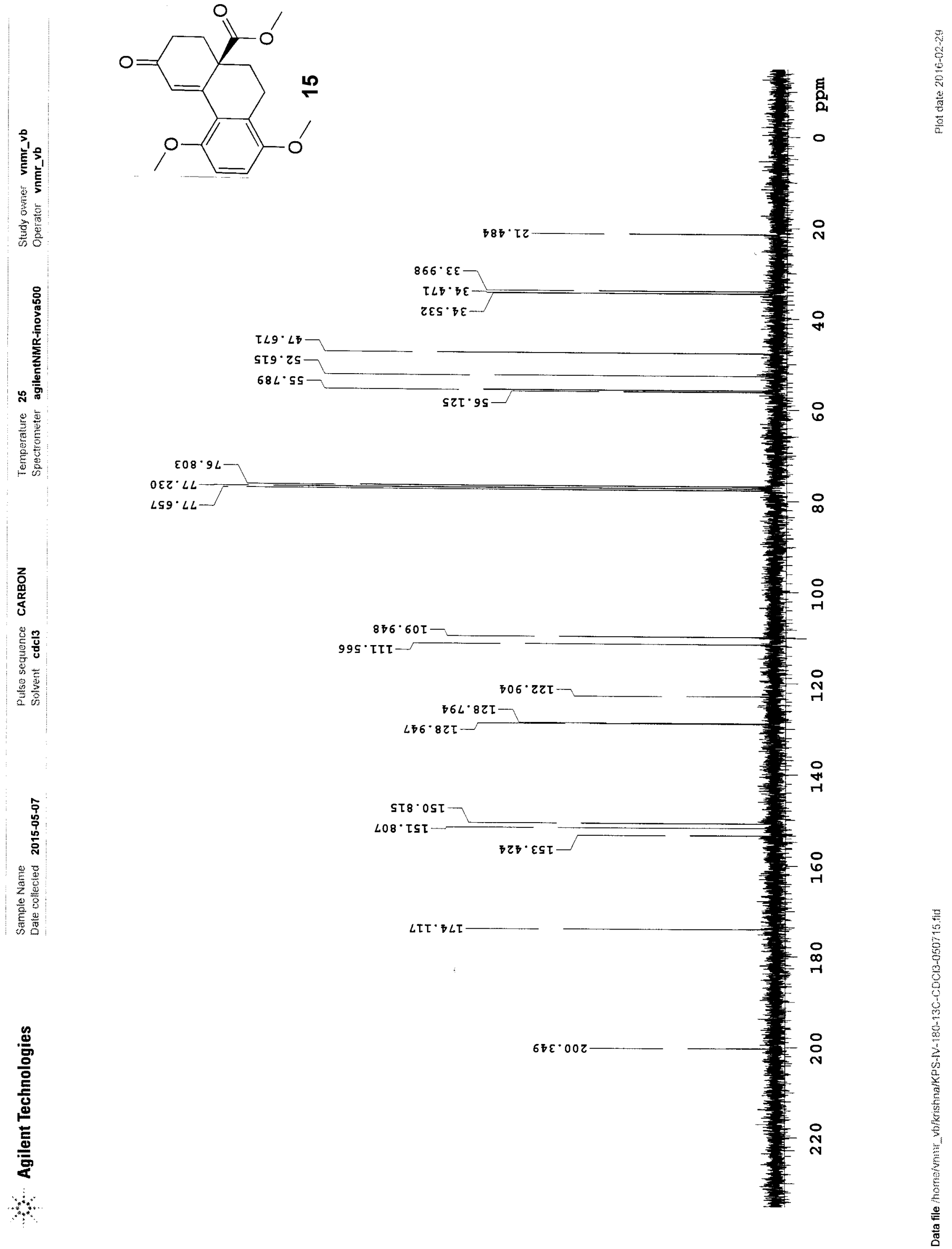

S-10 


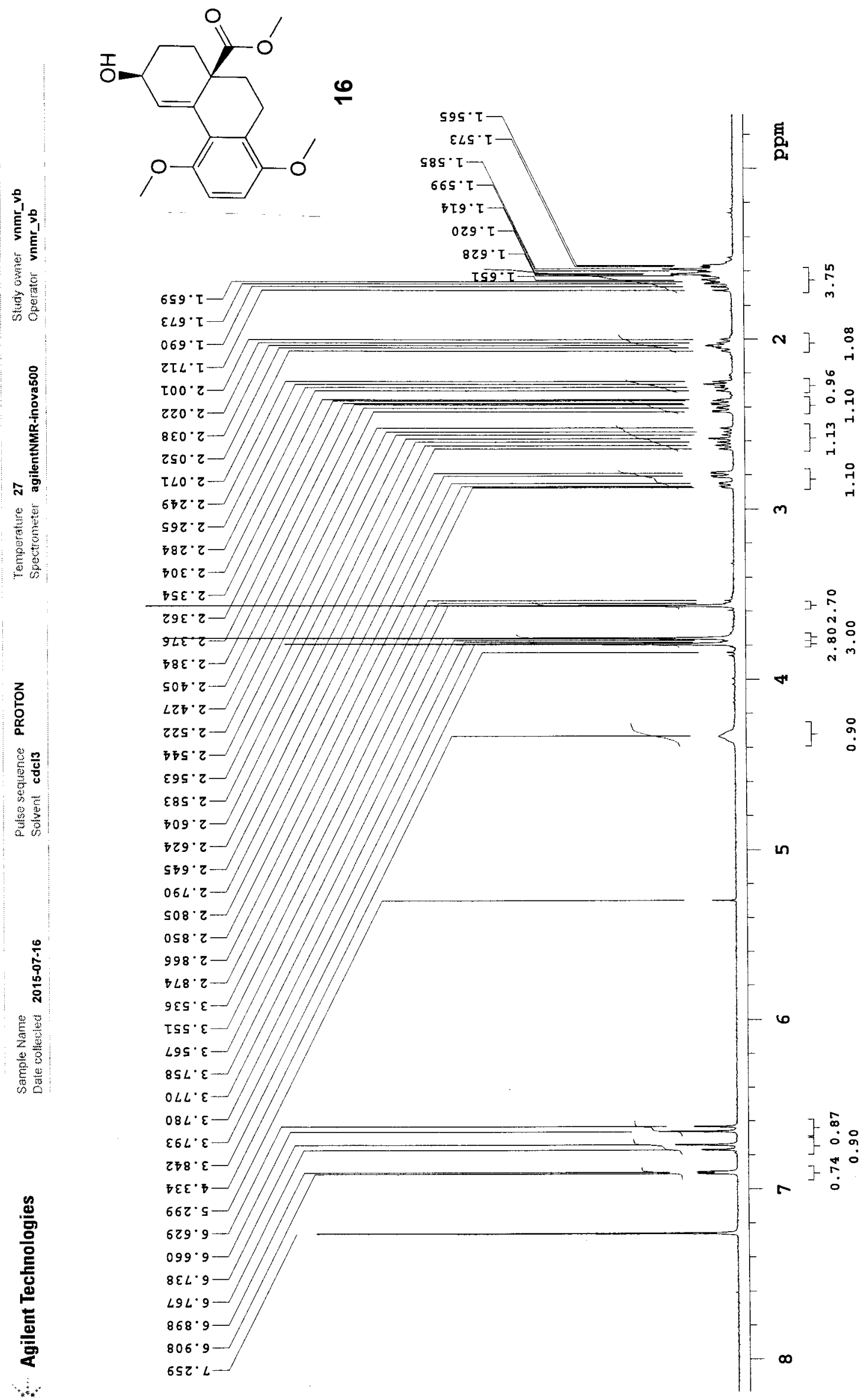




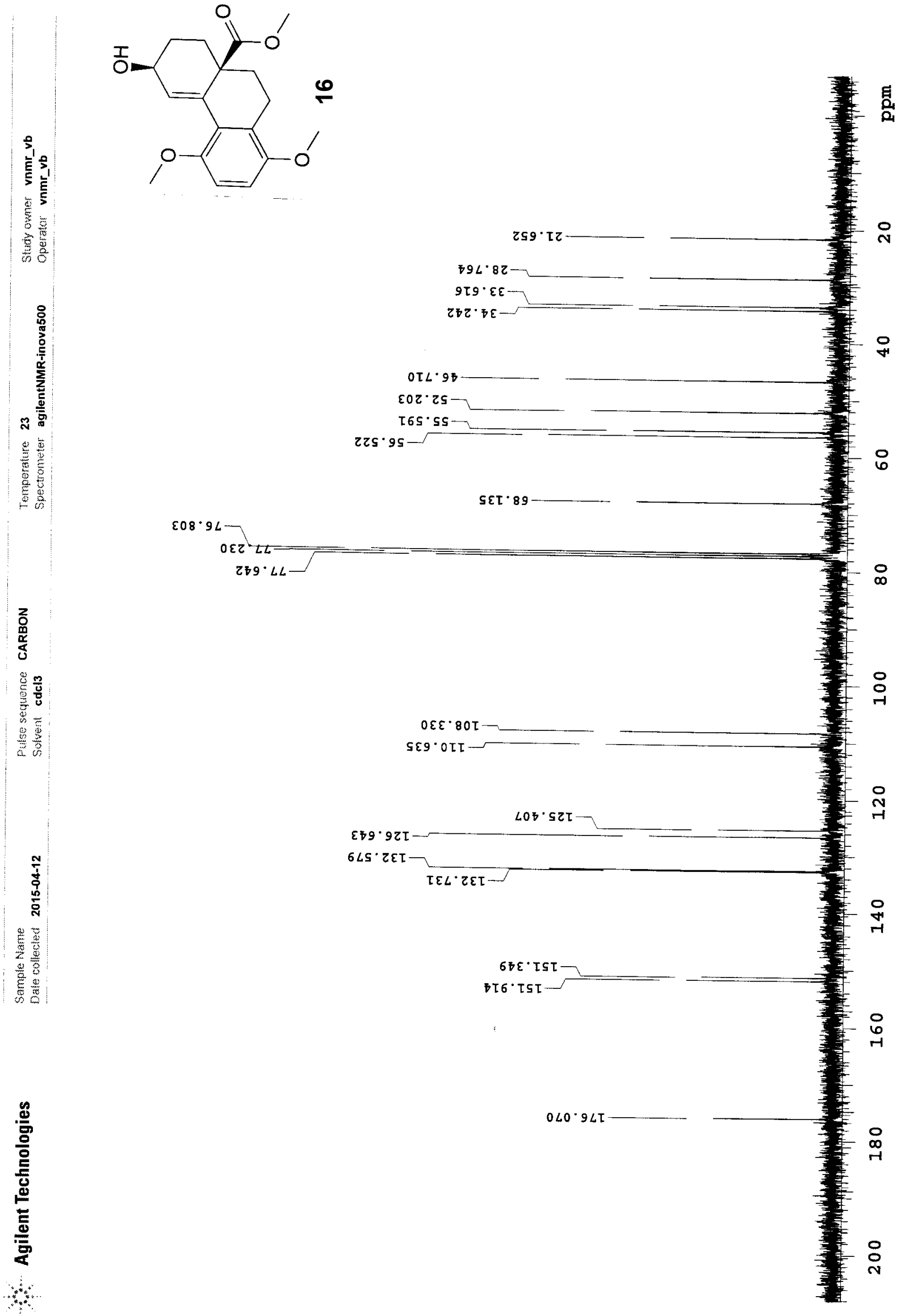




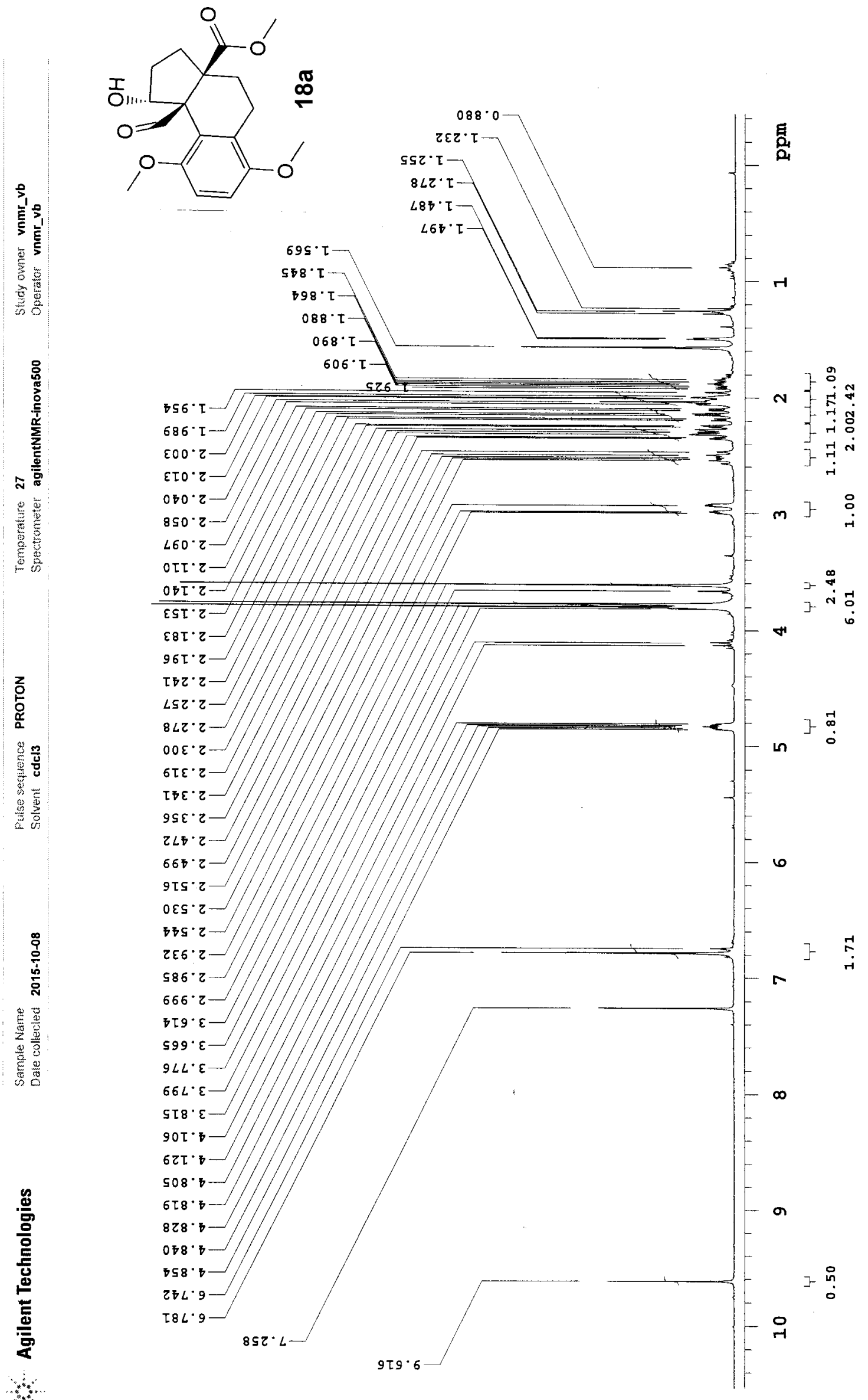




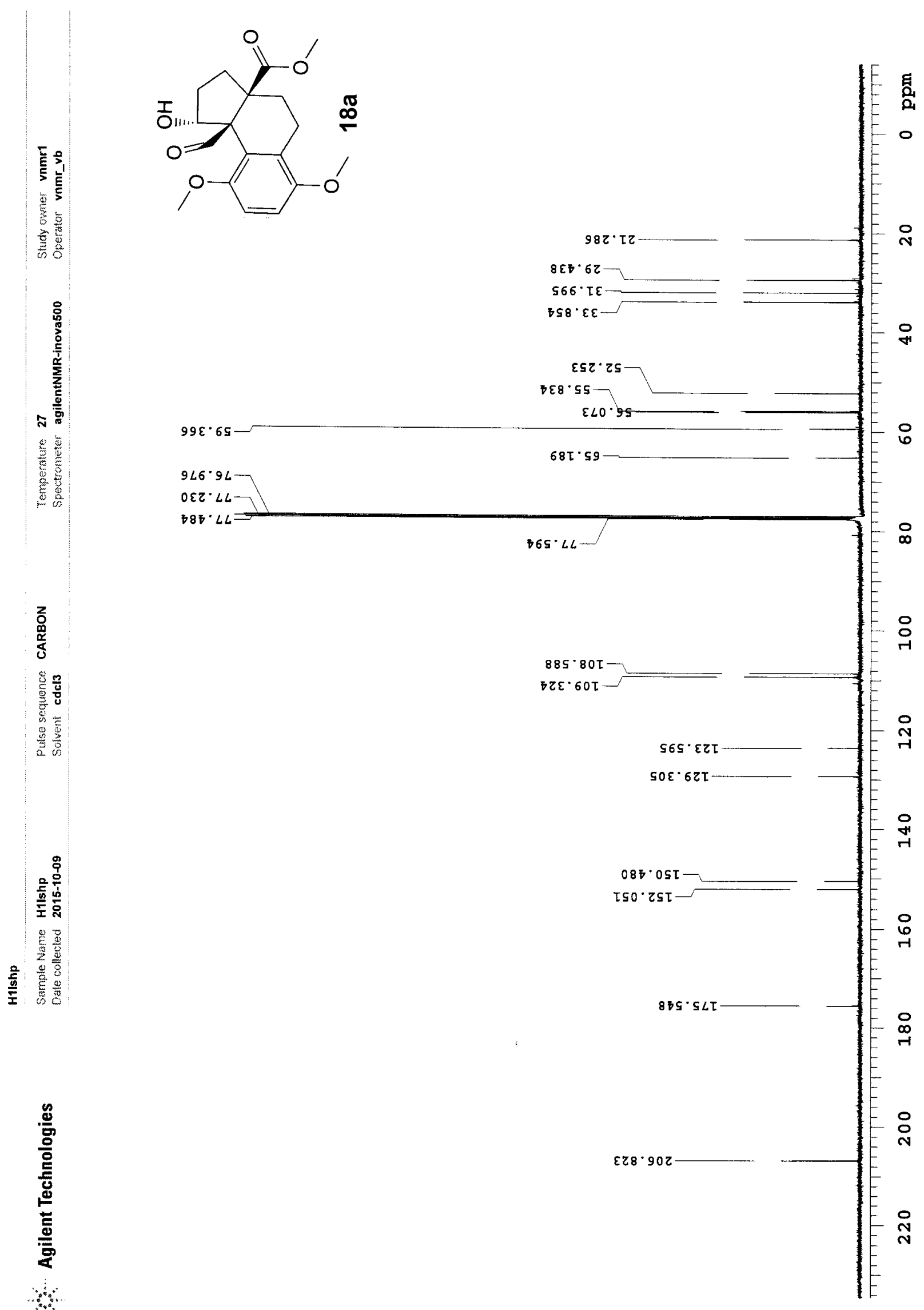


<smiles>COc1ccc(OC)c2c1CC[C@]13CC[C@@H](O)[C@@]21CCO3</smiles>

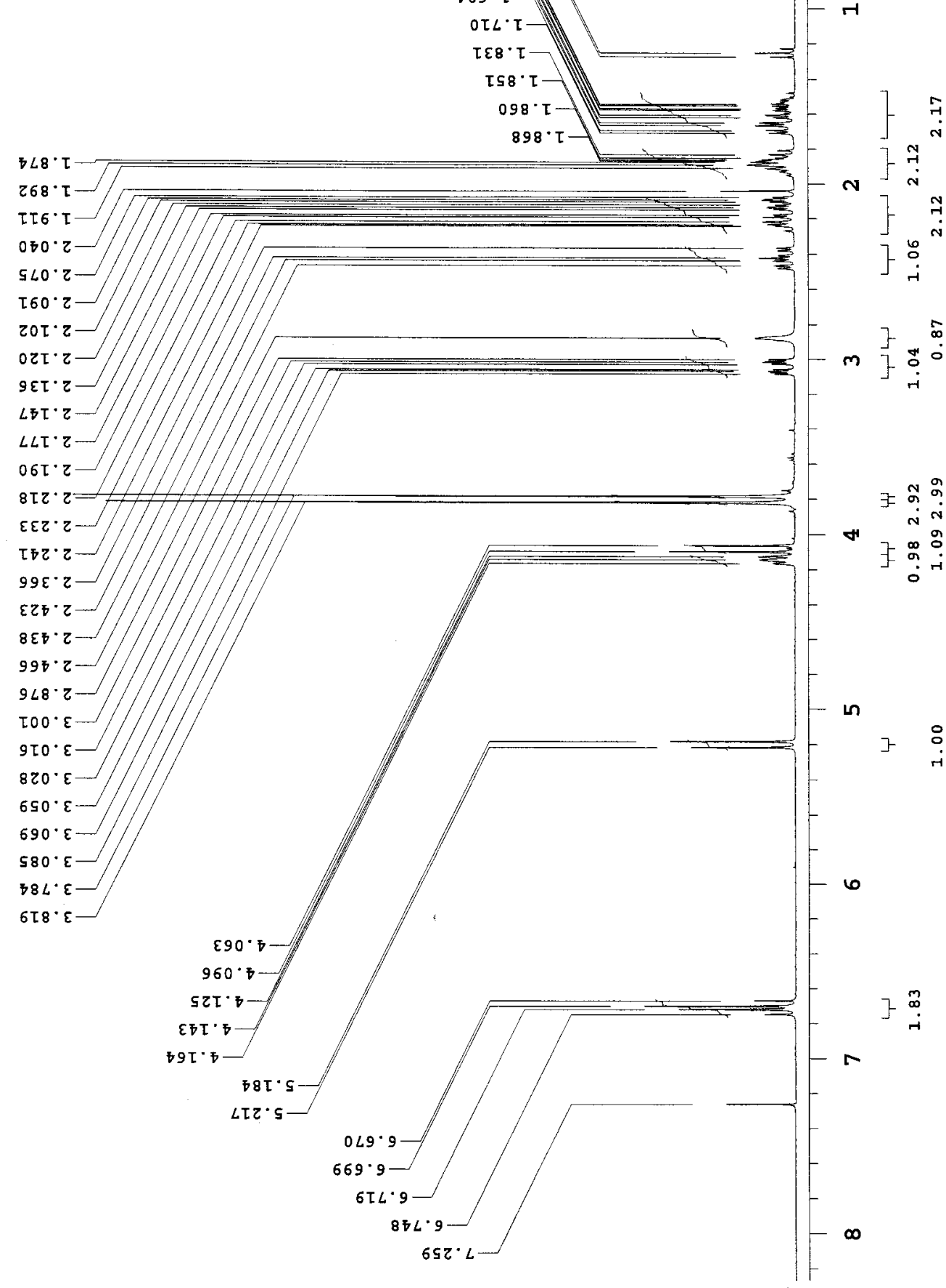




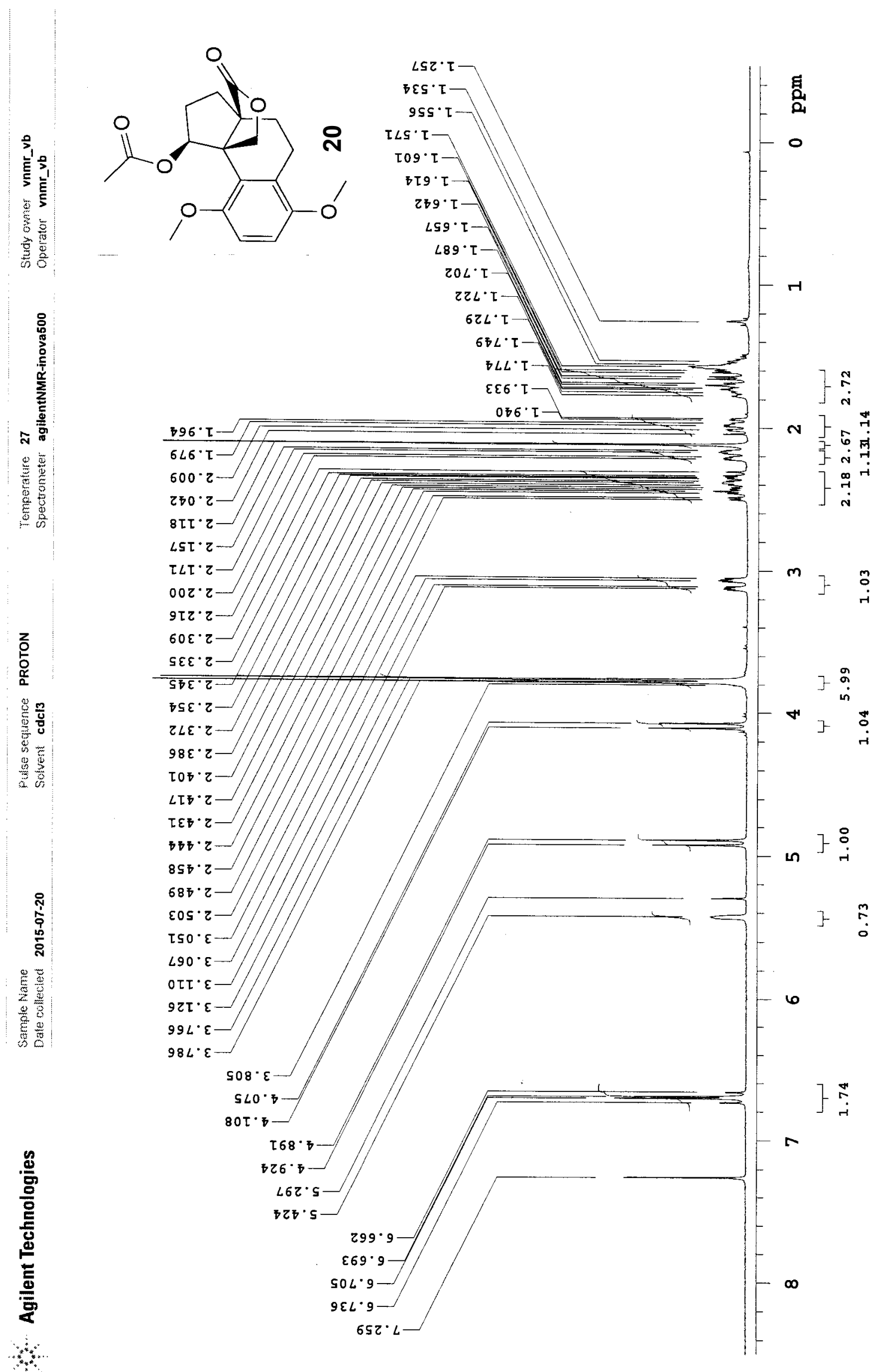



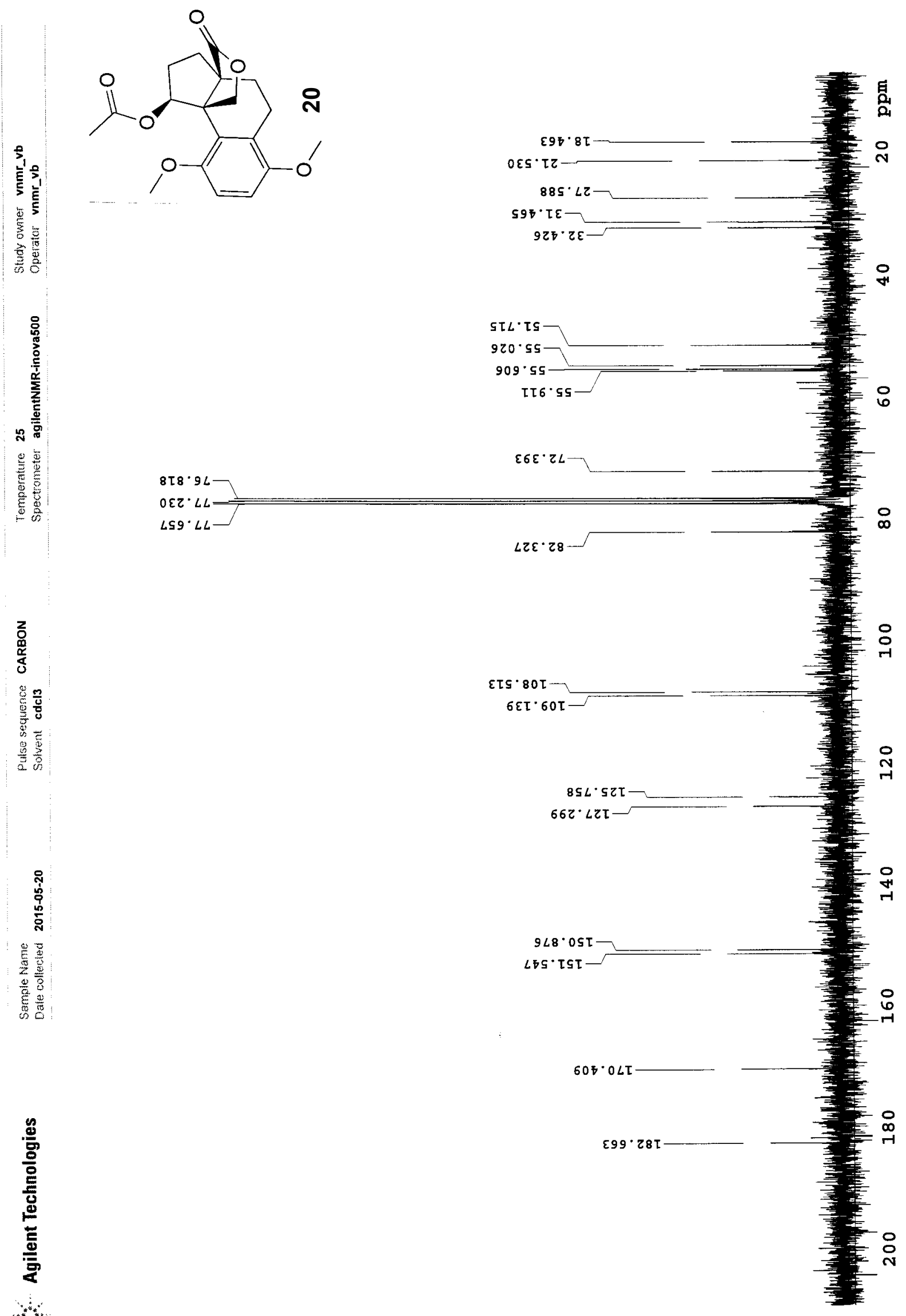


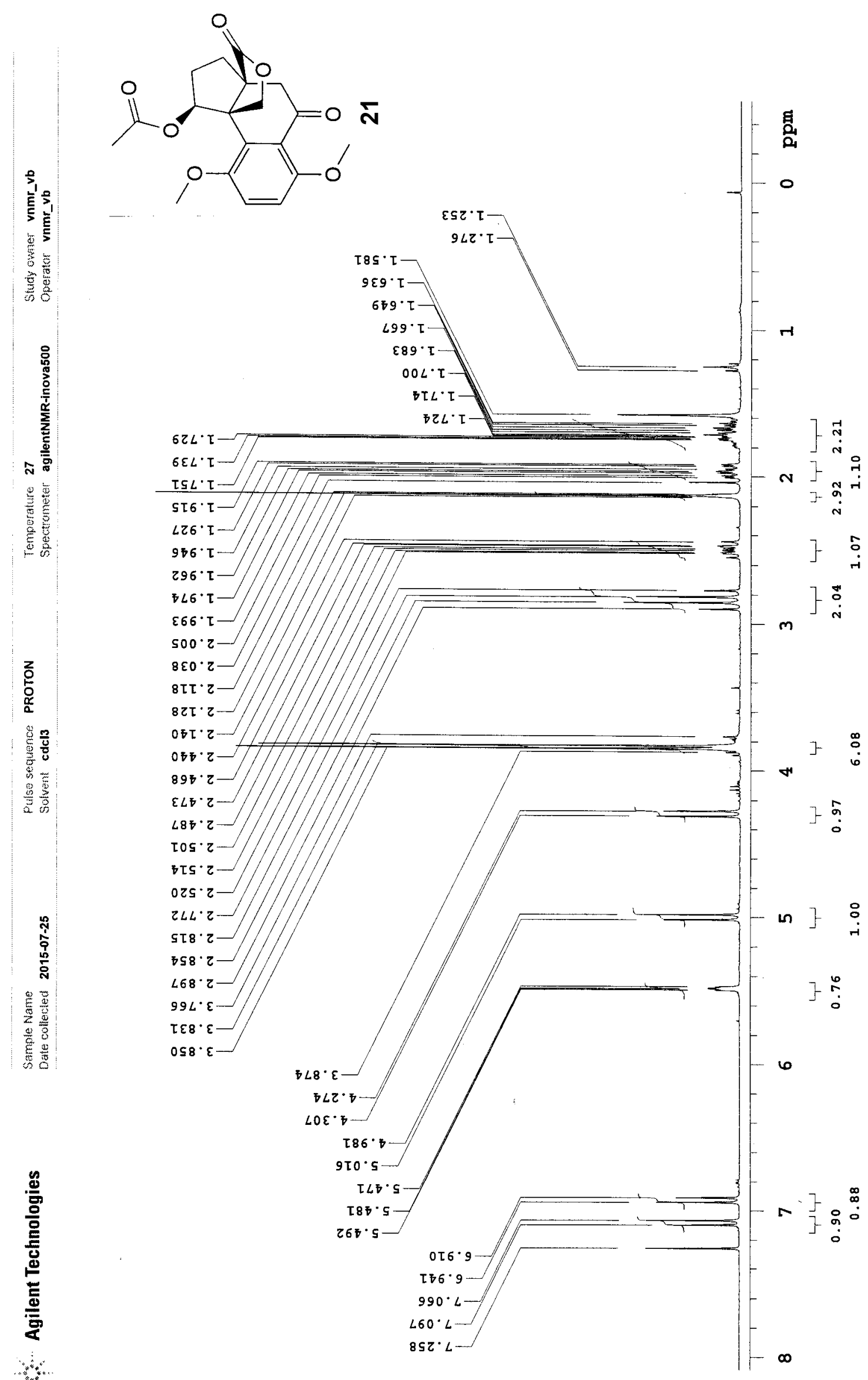




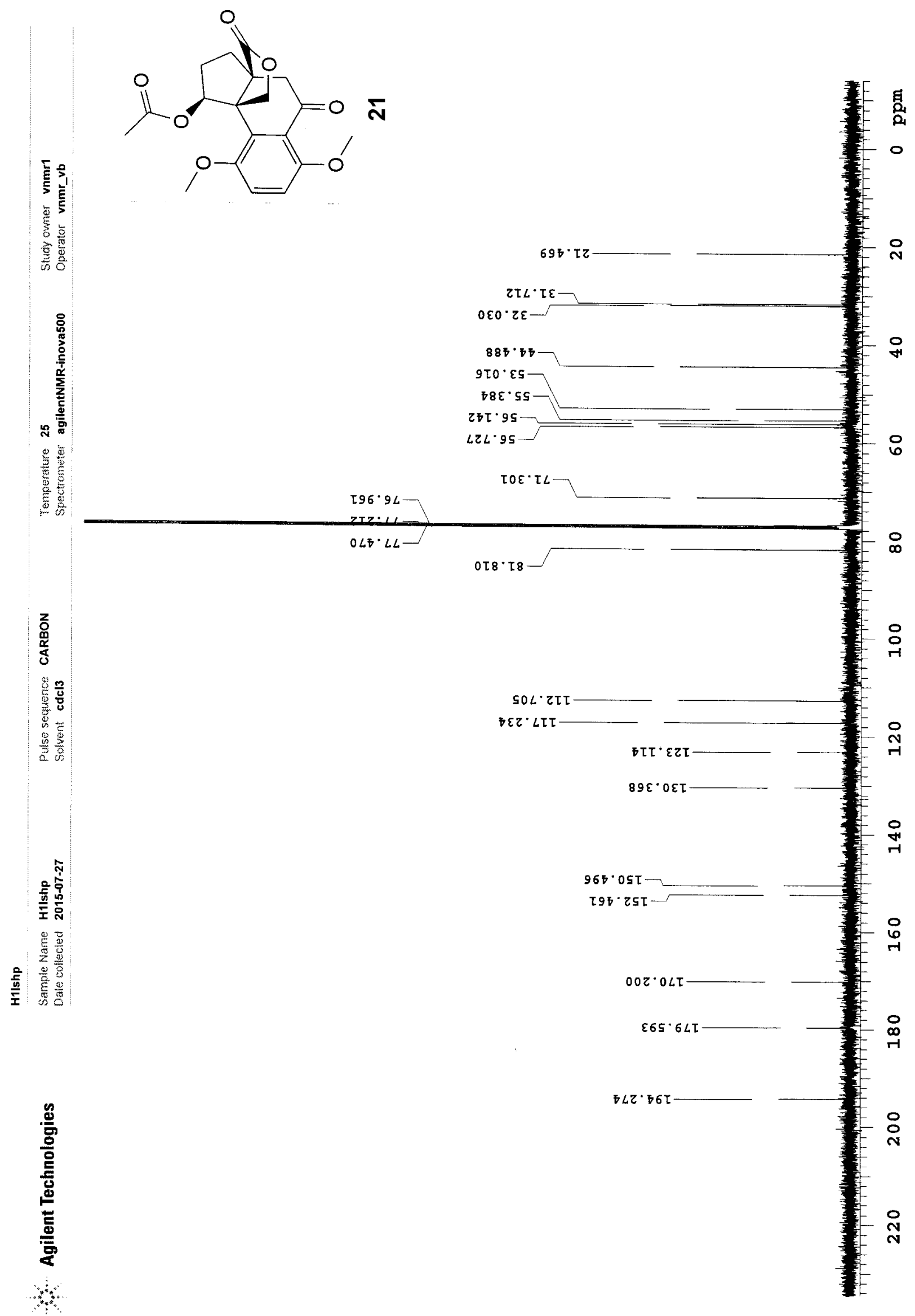




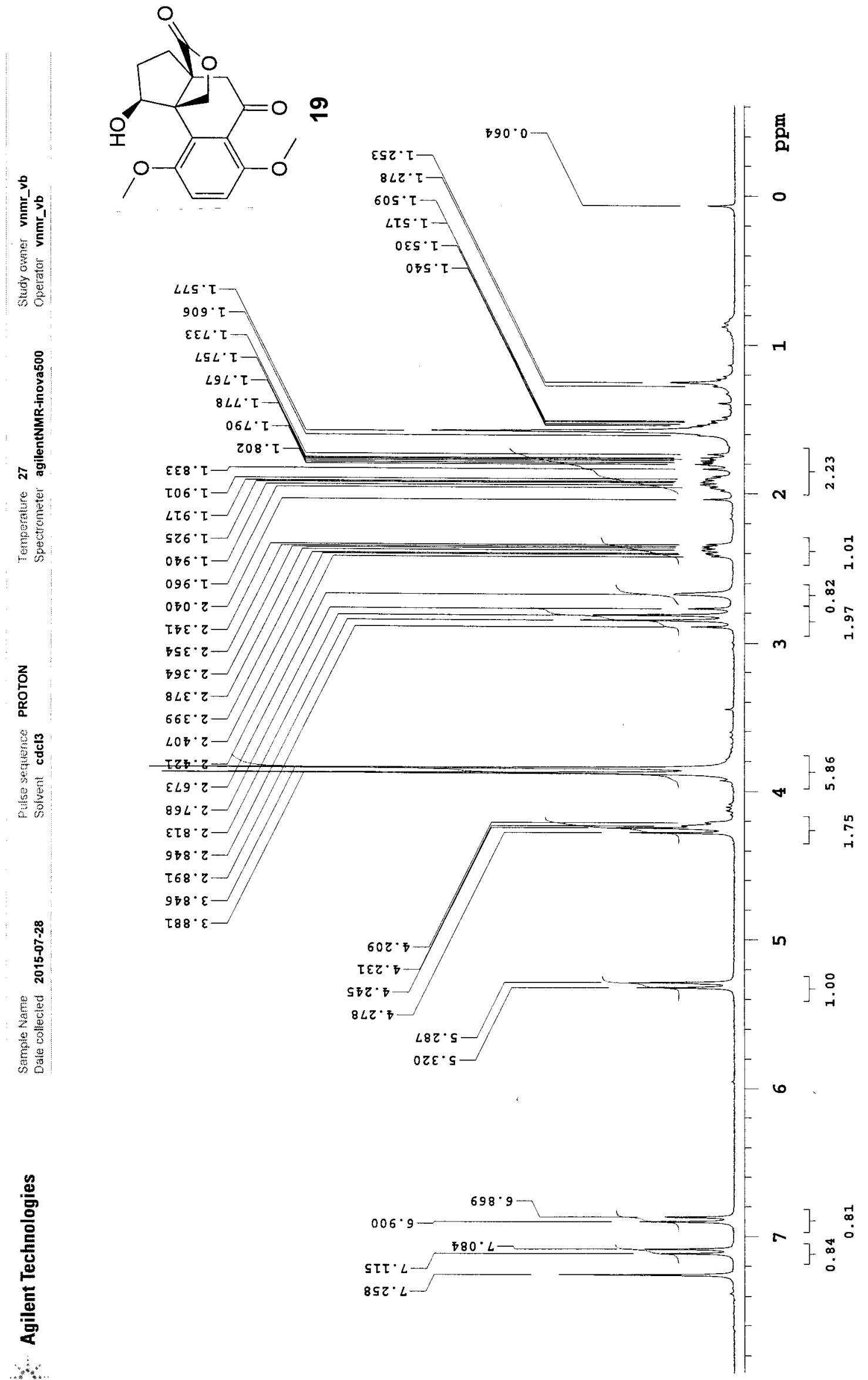




$$
1
$$




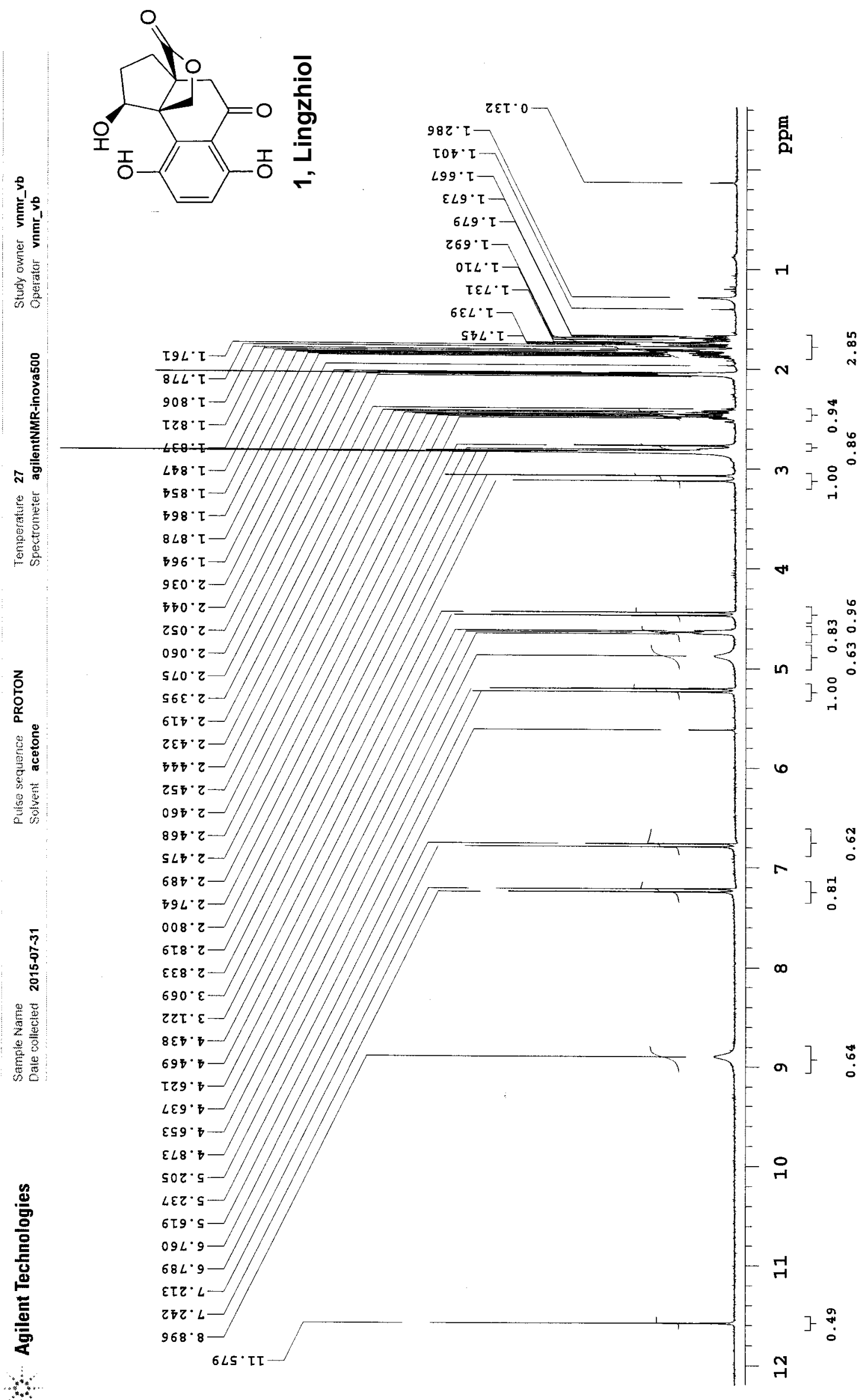




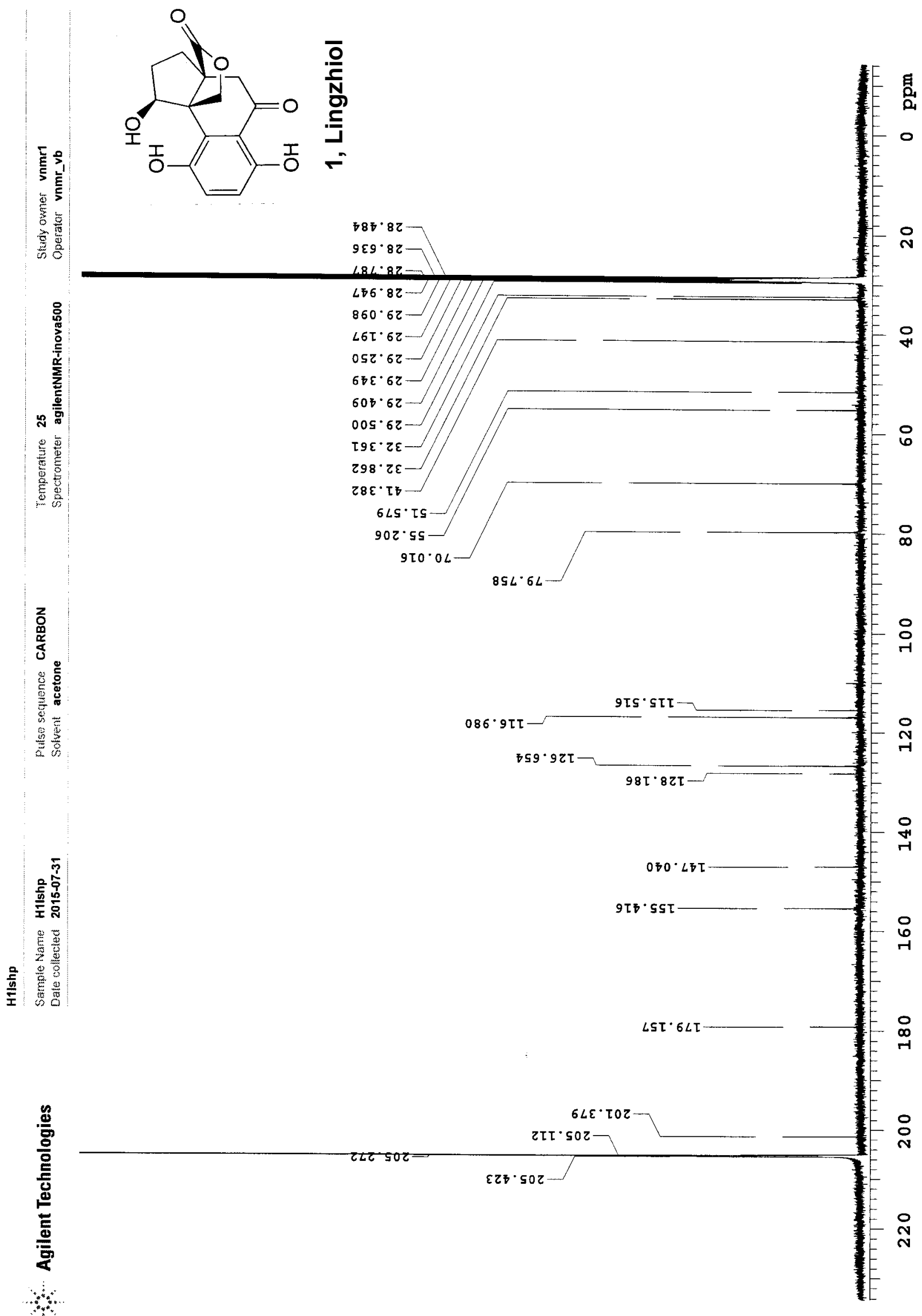

